\title{
Confirming acetylsalicylic acid hypersensitivity as a cause of chronic urticaria by desensitization
}

\author{
Katarzyna M. Puźniakowska, Marika Gawinowska, Marta Chełmińska
}

Department of Allergology, Medical University of Gdansk, Gdansk, Poland

Adv Dermatol Allergol 2019; XXXVI (3): 374-375

DOI: https://doi.org/10.5114/ada.2019.85645

A female aged 72 , with diabetes type 2, hypertension, hypercholesterolemia, narrowing of the left internal carotid artery, suspected coronary heart disease, nodular thyroid disease with subclinical hyperthyroidism, and osteoarthritis, was admitted to the Allergology Department due to chronic urticaria. In anamnesis, urticaria and angioedema started about 40 years before. At the beginning there was a correlation between symptoms and usage of nonsteroidal anti-inflammatory drugs (NSAID) (e.g. ketoprofen). There were also cutaneous changes after local use of ointments with analgesic drugs. Since September 2016 urticaria has been constant, sometimes with concomitant angioedema of eyelids. The patient observed worsening of symptoms after spicy meals. No additional symptoms indicating systemic reaction, like hypotension, dyspnoea, wheezing, gastrointestinal or other organ involvement were present. Aspirin (ASA) provocation was not suitable in this patient since she presented with constant urticaria. Thus, the basophil activation test with acetylsalicylic acid (BAT-ASA) was performed but the result was undiagnostic (due to unspecific degranulation of basophils without any factor). In December 2016, the patient was admitted to the Department of Allergology due to continuous severe urticaria. The patient was then treated with antihistaminic drugs and systemic steroids with good results, however an oral provocation test with acetylsalicylic acid could not be performed then. BAT-ASA was again undiagnostic (again due to unspecific degranulation of basophils without any factor). Even though after discharge the patient continued antihistaminic medication, recurrence of urticaria was observed.

The patient was again admitted to the Department of Allergology with generalized urticaria, without any other symptoms. During differential diagnosis of causes of chronic urticaria we excluded chronic infections (viral hepatitis, Lamblia infestation, parasites, Helicobacter pylori infection). Skin prick tests with aeroallergens and food allergens were negative. Treatment of hypertension did not contain any medication commonly known for causing urticaria. Spirometry was normal. Because of narrowing of the internal carotid artery the patient used ASA (75 mg/day), which the patient did not mention during her previous hospital stay. Aspirin was discontinued, dexaven was given, and low salicylate diet was applied. New urticarial skin changes appeared daily and antihistaminic medication was added. Performing an oral challenge with salicylic acid was impossible. In peripheral blood there was no basophiles so degranulation test would be again undiagnostic. After discussing her clinical situation with the patient, even though there was no confirmation of aspirin sensitization, we decided to perform desensitization with acetylsalicylic acid. No side effects of desensitization were observed, urticarial skin changes stopped appearing. A dose of $600 \mathrm{mg}$ was safely achieved and then a therapeutic dose of $300 \mathrm{mg} /$ day was continued. After discontinuation of antihistaminic drugs and systemic steroids, no urticaria was observed. This confirms diagnosis of acetylsalicylic acid sensitization as a cause of urticaria and angioedema. After 3 months the patient still did not need any antihistaminic medication, had normal diet, and no urticaria was observed.

The gold standard of diagnosing hypersensitivity to acetylsalicylic acid is an oral blinded challenge. The positive result of challenge confirms diagnosis of aspirin hypersensitivity. However a few conditions must be fulfilled, including discontinuation of antihistaminic drugs and systemic steroids, further, the patient should be asymptomatic at the time of test performance. If for medical reasons discontinuation of drugs is impossible or there are any contraindications for oral challenge, BAT-ASA can be used. Sensitivity of BAT-ASA is $37 \%$ and specificity $90 \%$, so the negative result cannot exclude diagnosis of hypersensitivity and oral challenge should be performed. In the presented case the patient had continuous urticaria with necessary usage of systemic steroids. Oral challenge could not be performed. BAT-ASA was twice undiagnostic and lack of basophiles excluded performing this test again. Due to lack of other reliable

Address for correspondence: Katarzyna M. Puźniakowska MD, Department of Allergology, Medical University of Gdansk, 7 Dębinki St, 80-952 Gdansk, Poland, phone: +48 5858443 00, e-mail: kpuzniakowska@wp.pl

Received: 8.11.2017, accepted: 5.04.2018. 
methods of diagnosing ASA hypersensitivity and significant discomfort of the patient caused by chronic urticaria we discussed desensitization without confirmation of ASA hypersensitivity. The patient was informed about the clinical situation, standard protocols and difficulties observed in this case. She decided to start desensitization protocol. Given the disappearance of skin changes during desensitization and a long-lasting effect (lack of relapses during next 3 months), we can acknowledge that suspected acetylsalicylic acid hypersensitivity was a correct diagnosis [1-4].

\section{Conflict of interest}

The authors declare no conflict of interest.

\section{References}

1. Niżankowska-Mogilnicka E, Bochenek G, Mastalerz L, et al. EAACI/GA2LEN guideline: aspirin provocation tests for diagnosis of aspirin hypersensitivity. Allergy 2007; 62: 1111-8.

2. Sánchez-Borges M, Caballero-Fonseca F, Capriles-Hulett A, González-Aveledo L. Hypersensitivity reactions to nonsteroidal anti-inflammatory drugs: an update. Pharmaceuticals 2010; 3: 10-8.

3. Abuaf N, Rostane H, Barbara J, et al. Comparison of CD63 upregulation induced by NSAIDs on basophils and monocytes in patients with NSAID hypersensitivity. ) Allergy (Cairo) 2012; 2012: 580873.

4. Lee J. Aspirin desensitization as a treatment for aspirin-sensitive chronic spontaneous urticaria. Dermatol Ther 2015; 28: 4-6. 